\title{
New Proposed Architecture For Q3 INTERFACE TO MANAGE IP-BASED NETWORKS
}

\author{
Gholamreza Farahani \\ Department of Electrical Engineering and Information Technology, Iranian Research \\ Organization for Science and Technology (IROST), Tehran, Iran
}

\begin{abstract}
This paper presents a new architecture of Q3 interface to combine OSI and Internet management protocols CMIP and SNMP with union management protocol in Telecommunication Management Network (TMN). To manage both OSI and IP-based networks, new proposed sub-layer is added between upper layers (SNMP and CMIP) and lower layers stack protocols such that with use of this sub-layer the TMN is able to communicate data between CMIP and SNMP with OSI and TCP/UDP/IP. Results of our simulation are shown that our new architecture named SUL (Sub-layer between Upper and Lower layers) has better performance than CMIP with $12.5 \%$ improvement in M-Get and M-Set services and $25 \%$ in M-Event Report service. Also SUL architecture performance will overcome to SNMP with $125 \%$ in M-Get and M-Set services and $66 \%$ in M-Event Report service. Usage of Q3 interface in telecommunication management network will improve the performance of instruction interchange approximately $26.6 \%$ in comparison to ordinary telecommunication.
\end{abstract}

\section{KEYWORDS}

Transmission Control Protocol, Internet Protocol, User Datagram Protocol, Simple Network Management Protocol, Common Management Information Protocol.

\section{INTRODUCTION}

Nowadays usage of computer networks is growing up in different applications. Further, the network architectures are very complex and diverse. With this growth of computer networks, requirement for a high level of service and performance is necessary. It is important to capable to propose a union management architecture, which covers a range of services, protocols and geographical borders. Reference [1] examines such network management infrastructures and addresses key issues such as TCP/IP network management, as well as issues pertaining to TMN, Telecommunications Information Networking Architecture (TINA), Telco and Corporate networks also further examines state-of-the-art technologies such as: managed objects, SNMPv3, CMIP, Q3 interfaces, TMN (Telecommunications Network Management), TINA and policybased management.

Management of networks has related to some activities like as design, presentation and control of network resources. Because of variety of network resources, management of them is complicated. As reference [2], internet is working based on Simple Network Management Protocol (SNMP) that is designed to manage the network simply, while management protocol named Common Management Information Protocol (CMIP) is designed for managing the OSI model. There are some advantages for SNMP like as simplicity and fast run time of it. Therefore usage of SNMP grows up rapidly. For implementation of CMIP and SNMP, always there are difficulties such as implementation of CMIP over TCP/UDP/IP (CMOT) and SNMP over OSI [3]. Because of networks variety, there are different private interfaces in them, thus to manage each private 
International Journal of Computer Networks \& Communications (IJCNC) Vol.9, No.4, July 2017

network, different interfaces is required to operate the protocols in these networks. Usage of internet in the worldwide is grown up, therefore for information interchange, internet mode profile against connection oriented and connectionless mode profiles will use as a global method in the world, which it consists of TMN [4].

To clarify the duty scope of each part of TMN, management architecture with standard connection interface should be used. Therefore standard institute ITU-T declared first standard for TMN in 1985. TMN may be considered as a concept in the discrete network, which has some connections at different points. These connections are shown in figure 1 [5]. According to figure 1 , telecommunication network has connection points with TMN at switches and transmission systems. Table 1 shows the required recommendation of the protocols which implement internet profile in each layer [6].

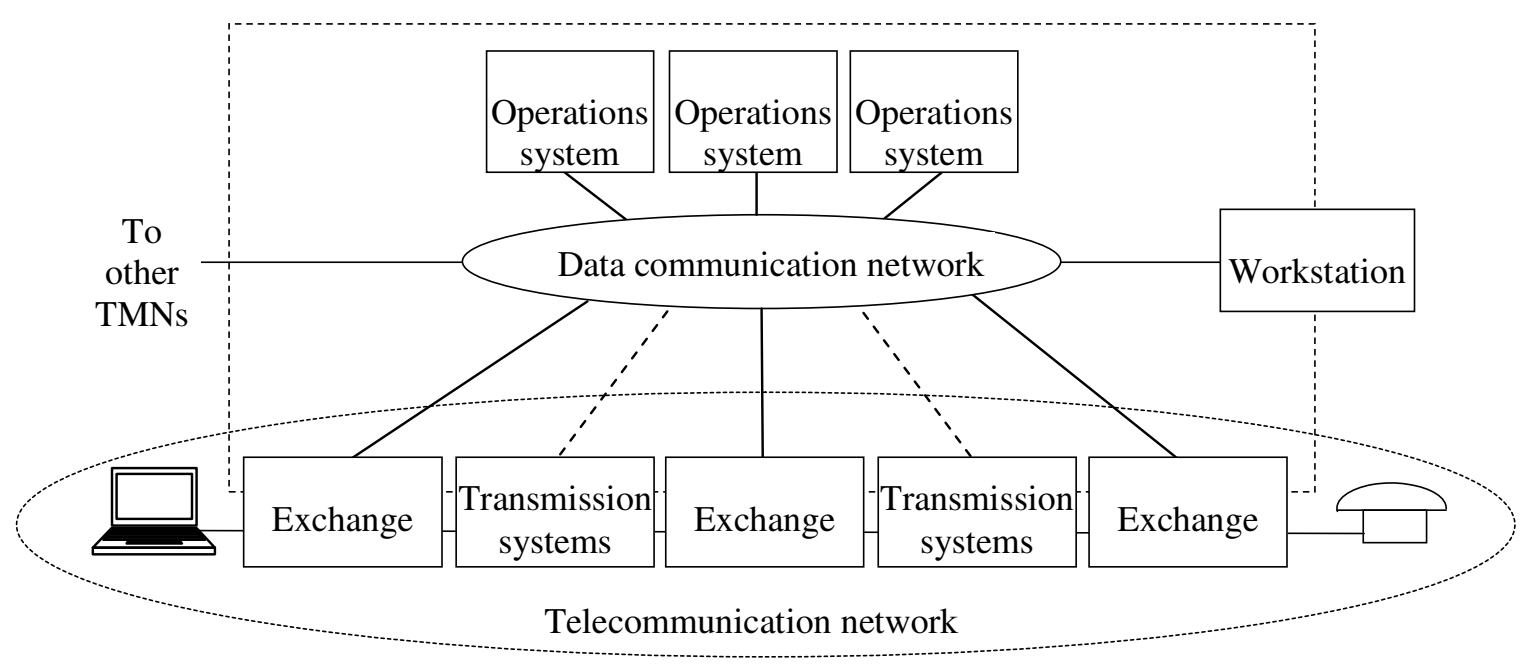

Figure 1. Relation between TMN and telecommunication network.

Table 1. Internet profile protocols in each layer with their recommendations

\begin{tabular}{|l|l|}
\hline Layer Name & Recommendation \\
\hline Transport layer & STD 0035 (RFC 1006), TCP STD 0007 (RFC 0793) \\
\hline Network layer & IP STD 0005 (RFC 01, RFC 0950, RFC 0919, RFC 0922, RFC 0792, RFC 1112) \\
\hline Data link layer & Sub-network access Protocol (not specified) \\
\hline Physical layer & Not specified \\
\hline
\end{tabular}

Most producers of telecommunication equipment currently are substituting their profiles with internet mode profile, for compatibility with data transmission systems in the base of TCP/IP. SNMP has been designed for all-purpose management protocols. The main point in the SNMP design is simplification of management operations as much as possible, therefore usage of it on each inter-network system has been successful and its operation will not stop easily. Although this SNMP has an advantage of simplicity in network management, it has some weakness such as this protocol has not powerful functions. Figure 2 shows the management network model for TCP/UDP/IP networks [5, 7]. 
International Journal of Computer Networks \& Communications (IJCNC) Vol.9, No.4, July 2017

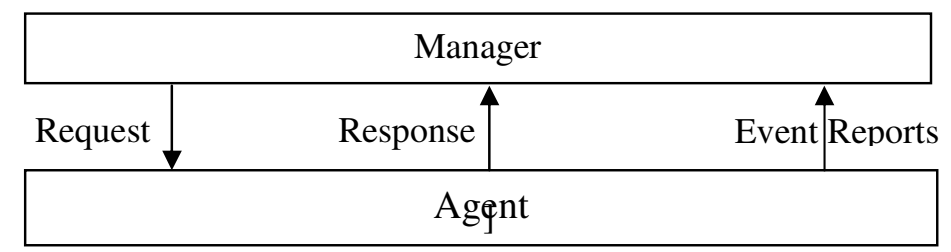

Figure 2. Manager-Agent model for TCP/UDP/IP networks.

The components of management model are Manger entity, Agent entity, Management Information Base (MIB) and Management Protocol between two entities (SNMP). Design of Management protocol for the simple network should be such that it contains application levels. For implementation of SNMP at fourth layer of network, User Datagram Protocol (UDP) will use. UDP is a connectionless protocol and SNMP will sit over UDP, hence SNMP is also connectionless. Manager entity may send three management messages towards the agent entity according to the conditions which these messages are Get Request, Get Next Request and Set Request. Agent entity will response to these messages with Get Response service. Figure 3 shows SNMP architecture [6].

\begin{tabular}{|c|c|c|}
\hline Managed application & \multirow{6}{*}{$\underset{\text { Messages }}{\stackrel{\text { SNMP }}{\longrightarrow}}$} & Managed application \\
\hline SNMP Manager & & SNMP Manager \\
\hline UDP & & UDP \\
\hline$\overline{I P}$ & & IP \\
\hline Data Link Layer & & Data Link Layer \\
\hline Physical Layer & & Physical Layer \\
\hline
\end{tabular}

Figure 3. SNMP architecture.

OSI management structure is similar to SNMP and its components are manager entity, agent (managed) entity, management connection protocol CMIP and Management information base [5, 8]. CMIP characteristic implicates a precise protocol that includes connect, disconnect and sending management messages.

Figure 4 shows network layers of CMIP with details of application layer which are System Management Application Service Element (SMASE), Common Management Information Service Element (CMISE), Association Control Service Element (ACSE) and Remote Operation Service Element (ROSE). User will use the ACSE element to connect and disconnect the link. At CMIP two condition of connection-oriented and connectionless have been considered.

SMASE element includes some System Management Functions (SMF) and its structure is different in comparison with other elements. SMASE element doesn't have clear rules and standard. Some samples of these functions are: Alarm report, Alarm declaration, Alarm abstracting and etc.

ROSE functional element will use to send a request toward the remote system and receive its response. In spite of SMASE element, in Rose element there is a standard structure for sending request and receiving its response. 
International Journal of Computer Networks \& Communications (IJCNC) Vol.9, No.4, July 2017

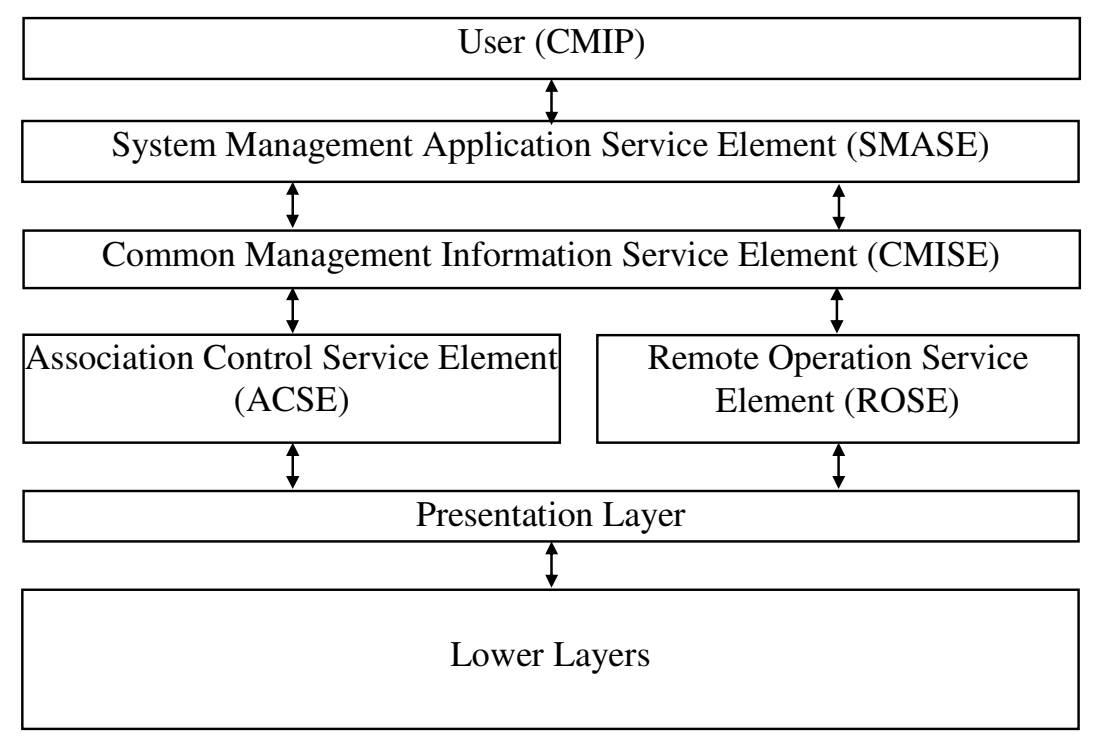

Figure 4. Management structure of network layers at CMIP.

CMISE element provides services and protocols to better clarify the request and its response structure in cooperation with ROSE element. With usage of the services and protocols of CMISE element, all management information will pass to the ROSE functional element and the result will send. Different models could implement on the IP network protocol $[9,10]$ but in this paper we focus on the increasing performance of IP-based networks.

In the recent years some improvement is carried out of the network architecture named Next Generation Network (NGN) architecture. In Reference [11] several end-to-end Quality of Service (QOS) for NGN (called NGNQOS) are proposed that are

- Presentation of the application layer to support Session Initiation Protocol (SIP)

- Preparation of network control layer to overcome the blockage problem of edge nodes or servers for end-to-end admission control

- Usage of adaptation layer to support different network configurations and mobility

- Use of network transmission layer to prepare end-to-end QOS controls for real-time communications with integrating DiffServ and MPLS

- Provision of management layer that prepares web-based client-server Graphical User Interface (GUI) browser and wireless information connection.

Current network management technologies follow two approaches: ITU-T's recommendations for TMN and IETF's SNMP that favours IP networks. The applications based on these methods are specifically targeted at the variety of independent networks. These networks are Public Switched Telephone Network (PSTN), the Public Switched Data Network (PSDN), the Public Land Mobile Network (PLMN), the Wireless Local Area Network (WLAN), and etc. All of these networks will be integrated into an infrastructure with single IP that will refer as the NGN in the near future. In reference [12] some emerging methods towards management of network in NGN are proposed.

The simulation for telecommunication switches is implemented in Iranian Telecommunication Research Center (ITRC). With using other functionalities of telecommunication management network in association with Q3 interface program, we would be able to configure our network in the optimal condition [13]. 


\section{DESCRIPTION OF TMN}

In TMN there are three different architectures. In this section these architectures will describe briefly.

\subsection{Functional Architecture of TMN}

In this architecture, five types of different functional architecture are defined which in implementation of TMN, all of them are not require. Figure 5 shows different types of functional blocks.

For description of the functional blocks in functional architecture of TMN, reference points will use. Therefore there are five sets of reference points. From these five reference points, three of them Q, F and X will define in TMN recommendation completely but two other reference points $\mathrm{M}$ and $\mathrm{G}$ are placed in the outside of TMN environment. In figure 6 reference points and their functional blocks are shown. At continuation of this subsection, functional blocks of TMN will describe briefly.

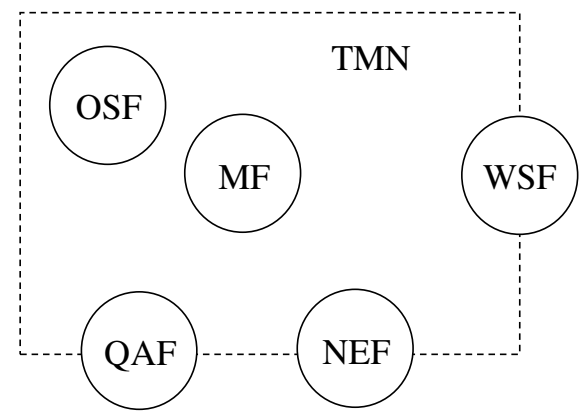

OSF Operations Systems Function

MF Mediation Function

WSF WorkStation Function

NEF Network Element Function

QAF Q Adaptor Function

----- The TMN functional boundary

Figure 5. Functional Blocks of TMN

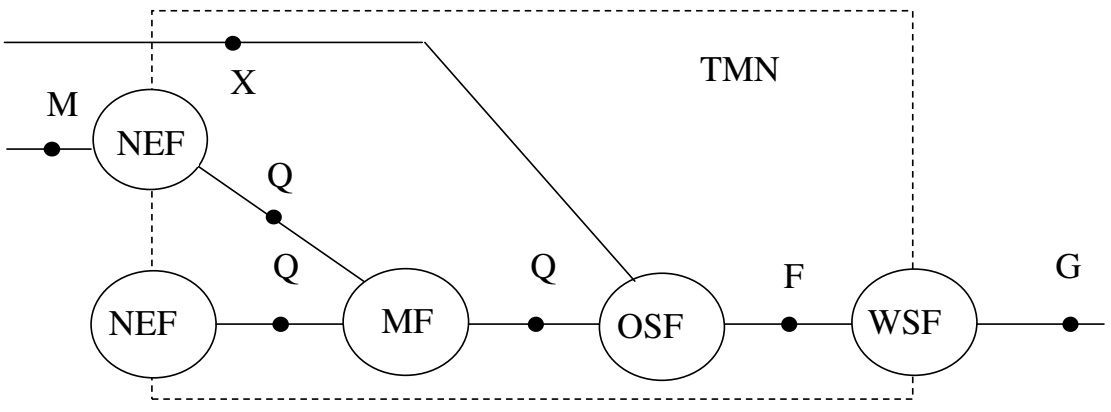

Figure 6. Different reference points in TMN 
International Journal of Computer Networks \& Communications (IJCNC) Vol.9, No.4, July 2017

\subsection{Information Architecture of TMN}

This architecture will discuss about interchange information structure between manager and agent. These information will use for management of information resources. Figure 7 shows the common schematic of relationship between two network management systems. Each management system includes manager, agent and information resources.

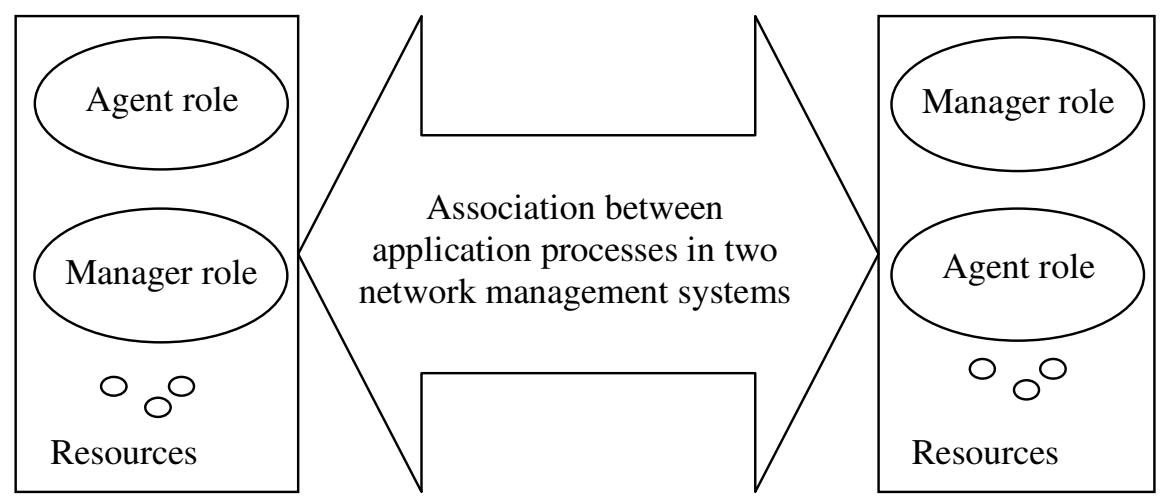

Figure 7. Common structure between manager, agent and information resources

\subsection{Physical Architecture of TMN}

In physical architecture of TMN, the implementation platform of TMN functional block with physical equipment will describe. Structural blocks usually implement functional blocks of their names. Table 2 shows functional and structural blocks relation [2].

Table 2. Relation between functional and structural blocks

\begin{tabular}{|c|c|c|c|c|c|}
\hline & NEF & MF & QAF & OSF & WSF \\
\hline NE & M & O & O & O & O* \\
\hline MD & & $\mathrm{M}$ & $\mathrm{O}$ & $\mathrm{O}$ & $\mathrm{O}$ \\
\hline QA & & & $\mathrm{M}$ & & \\
\hline OS & & $\mathrm{O}$ & $\mathrm{O}$ & $\mathrm{M}$ & $\mathrm{O}$ \\
\hline WS & & & & & $\mathrm{M}$ \\
\hline DCN & & & & & \\
\hline
\end{tabular}

O: Optional

M: Mandatory

O*: May be exist if

OSF and MF exist 


\section{Q3 INTERFACE}

In $\mathrm{TMN}$, there are four different communication interfaces which are shown in table 3. As shown in table 3, Q3 interface includes different connections in TMN environment. Although Q3 interface uses to interact between different system functions but type of interchanged management information is different. The applications of Q3 interface in TMN are listed in front of Q3 in the table 3 which are between OS and NE, MD and NE, OS and OS in a TMN environment.

Table 3. Applications of different TMN interfaces

\begin{tabular}{|l|l|}
\hline Types of TMN interface & Application of interface \\
\hline X & Between OS and OS in different TMN environment \\
\hline F & Between WS and OS or WS and MD \\
\hline Qx & Between MD and NE \\
\hline Q3 & $\begin{array}{l}\text { Between OS and NE, MD and NE } \\
\text { Between OS and OS in a TMN environment }\end{array}$ \\
\hline
\end{tabular}

For Q3 interface, three different application classes are defined according to its requirements as below:

1. Interactive Class: This class deal with unexpected information that is received and must answer quickly.

2. File-oriented Class: This class uses for applications that information are in the management file.

3. Directory Class: When needs to map application entity and its address to establish the connection, this class will use.

\subsection{Upper Layers of Q3 Interface}

In this section necessary concept of upper layers protocol requirements will introduce, then according to the functions which supported with these layers, different services have defined [14].

\subsubsection{Functional Unit}

Functional unit is a technique to group the services into the intended sets. Based on services type with use of functional unit, interchange of information will carry out. 


\subsubsection{Application Service Element (ASE)}

In this element, specified function of application layer will carry out. ASEs may be utilized in different applications. When several ASEs are used at application layer, it is necessary to coordinate them which in this situation, coordination unit is necessary that includes functions to organize between ASEs.

Application Service Element consists of Remote Operation Service Element (ROSE), Association Control Service Element (ACSE), System Management Application Service Element (SMASE) and Common Management Information Service Element (CMISE).

\subsubsection{ROSE Functional Unit}

After connection establishment, with use of remote operation protocols, management information must be send to destination. Then destination with these protocols will send a response to the source. These requests and receiving related response will carry out with four services format in ROSE functional unit. These services are RO-INVOKE, RO-RESULT, RO-ERROR and ROREJECT.

To send initial request, the RO-INVOKE service will use. To send positive response to request from side of receiver, RO-RESULT service will utilize. For sending negative response to the request from side of receiver, RO -ERROR service will employ. To reject each of three services RO-INVOKE, RO-RESULT and RO-ERROR, the service RO-REJECT will use.

\subsubsection{ACSE Functional Unit}

ACSE functional unit will employ for connection-oriented services. This functional unit uses to establish and release the connection. The ACSE has four services which these services are AAssociate, A-Release, A-Abort and A-P-Abort.

A-Associate service will use for connection establishment. A-Release service is for gracefully disconnection. A-Abort service uses for suddenly disconnection by user of ACSE and A-P-Abort will utilize to suddenly disconnection by the provider (ACSE). Abstractly, ACSE services have a task of connection establishment and disconnection. Data transmission will carry out with services of other entities.

\subsubsection{SMASE Functional Unit}

For SMASE there is not clear protocol and standard in the implementation. This functional unit has several system management functions. These functions will employ for more clearance and transparency of information and parameters of CMISE. Hence SMASE in cooperation with CMISE will deliver complete information to ROSE [14].

\subsubsection{CMISE Functional Unit}

CMISE will utilize for common management information transmission between OS and NE. With use of services and protocols of CMISE, all of the common management information will transfer to ROSE unit and this information will send as a request or response. 


\subsubsection{Q3 Interface with TCP Protocol}

TCP is a connection-oriented protocol that has a few restrictions for lower layers for secure data stream transmission. Network layers schematic of TCP protocol is shown in Table 4.

Table 4. Network layers of TCP/IP protocol

\begin{tabular}{|c|}
\hline User or Upper layers \\
\hline TCP \\
\hline IP \\
\hline Connected Network or Lower Layers \\
\hline
\end{tabular}

As shown in table 4, TCP layer is seated on the IP. Because of the IP layer usage, TCP layer will able to send and receive information with fragments of different length [15]. One of the main features of the Internet Protocol is fragmentation of the TCP packets in the source and adhesive the fragments together in the destination. Also IP uses to transmit data and deliver it to the destination in several networks and gateways based on priority and security.

To implement TCP over IP, some parameters such as reliability, data stream control, multiplexing, congestion control, type of service, data communication, priority and security must be considered [10].

\subsubsection{TCP/ (Application or User) Interface}

The application layer at Q3 interface could connect to the network via TCP. TCP performs ACSE functional unit instructions and return their answers. All implementation of TCP has a minimum set of services to implement such as Open, Send, Receive, Close, Status, and Abort. Task of necessary services is as table 5 [16].

Table 5. TCP services

\begin{tabular}{|l|l|}
\hline Service Name & \multicolumn{1}{|c|}{ Description } \\
\hline Open & establish connection \\
\hline Send & send information \\
\hline Receive & receive information \\
\hline Close & graceful disconnection \\
\hline Status & $\begin{array}{l}\text { monitoring of connection status (includes external socket, local } \\
\text { connection name, receiving window, sending window, connection } \\
\text { status, number of buffers which are waited to receive ACK, number of } \\
\text { unknown buffers for urgent receiving, priority, security, fragmentation } \\
\text { and data transmission time) }\end{array}$ \\
\hline Abort & \begin{tabular}{l} 
abruptly disconnection \\
\hline
\end{tabular} \\
\hline
\end{tabular}




\subsection{OSI Model versus TCP/IP Model}

Table 6 shows the network layers of OSI and TCP/IP reference models. At TCP model, session and presentation layers are not exist and their works will carry out by transport and application layers respectively. Although both reference models have the application layer, but their services are different.

Transport layer at TCP/IP model, provide two standard protocol which named TCP and UDP. Network layer in the OSI model like as other models provides both connection-oriented and connectionless services while internet layer at TCP/IP model has only connectionless service. Connectionless Network Protocol (CLNP) at OSI model is like as IP protocol in performance and only its difference is that CLNP protocol has a variable address length while IP has a fixed address length [8]. Transport layer of TCP/IP doesn't have any restriction on the lower layers of this protocol but host should be capable to connect to the network and send its data with use of IP packet protocols.

Table 6. Comparison between OSI and TCP/IP models

\begin{tabular}{|l|l|}
\hline \multicolumn{1}{|c|}{ OSI } & \multirow{2}{*}{ TCP/IP } \\
\cline { 1 - 1 } Application (Layer7) & \multirow{2}{*}{ Application } \\
\cline { 1 - 1 } Presentation (Layer6) & \\
\hline Session (Layer5) & Transport \\
\hline Transport (Layer4) & Internet \\
\hline Network (Layer3) & \multirow{2}{*}{ Subnet } \\
\hline Data Link (Layer2) & \\
\hline Physical (Layer1) &
\end{tabular}

\subsection{Comparison CMIP with SNMP}

CMIP and SNMP, each of them has some advantages and weaknesses. Selection one of these protocols depends on the many parameters. Simplicity in the design is the main advantage of SNMP. Also when the communicated messages between manager and agent entities are low, SNMP will utilize. Low security is the main weakness of SNMP that recently is going to be better in the new versions. Because of using connectionless services in the transport layer, agent entity will not receive acknowledge, therefore it cannot sure that its alarm report reached to manager entity. Therefore when there is too much management information to communicate, the network traffic load rises and might be management information lost.

On the other hand, to compensate the lacks of SNMP, CMIP has designed and it can be used for lager networks. Object-oriented model will use to design and implement SNMP [17]. The main advantage of CMIP is its ability to define the techniques to cover manual control, security and filtering of the management information. The main weakness of CMIP is resource occupation time which is more than SNMP. Table 7 summarize the list of the CMIP and SNMP services. 
International Journal of Computer Networks \& Communications (IJCNC) Vol.9, No.4, July 2017

Table 7. CMIP and SNMP services

\begin{tabular}{|l|l|l|}
\hline \multicolumn{1}{|c|}{ Name } & \multicolumn{1}{c|}{ Valid Service(s) } & \multicolumn{1}{c|}{ Description } \\
\hline Get & CMIP and SNMP & Obtain a value maintained by the managed object. \\
\hline Set & CMIP and SNMP & Set a value maintained by the managed object. \\
\hline Event Report & CMIP and SNMP & Report special conditions about a managed object. \\
\hline Get Next & SNMP & $\begin{array}{l}\text { Name and value of the next SNMP attribute will determine } \\
\text { in the object. }\end{array}$ \\
\hline Action & CMIP & $\begin{array}{l}\text { One of the actions defined for the managed object will } \\
\text { invoke. }\end{array}$ \\
\hline Create & CMIP & Instance of an object class will create. \\
\hline Delete & CMIP & Delete an instance of class. \\
\hline
\end{tabular}

\section{IMPLEMENTATION}

Regards to client-server property in TCP/IP protocol and using TCP/IP at lower layers of Q3 interface; implemented software has a client-server property. TCP/IP protocol is connectionoriented; therefore when one client connects to the server, a portion of server time will allocate to receive information from client, even if client sends its information with short time gap. Hence during the answering or listening of server to the client, other requests of clients to the server must be queued until connection between a client and server released and server able to answer another client request which is time-consuming. To overcome this problem, multithreading feature of programming is used in the software [18]. Socket programming is proper feature of programming to create relation between application programs and TCP/IP protocol. In socket programming, input arguments will give to the each socket and socket will return one or more results which these results will utilize as an end point to establish connection in TCP/IP.

To implement and calculate simulation results, instead of telecommunication switches, client computers are used to simulate them, and server computer simulate control switch to make a required instructions for controlling telecommunication switches. Simulation of client-server network, functional test program for send/receive, connect/disconnect and other functions of TCP/IP protocol are programmed with visual $\mathrm{C}++$ software [19]. According to received information from application layer, connection conditions such as establishment and release will carry out. Also clean and unaccepted data sending and different states of TCP were implemented in the simulation software [20].

\subsection{Proposed Architecture to Manage Network}

As explained in subsection 3.3, each of the CMIP and SNMP might implement on the OSI and TCP/UDP/IP models separately. In the proposed architecture, the main advantage is the possibility of network management of both OSI model and TCP/UDP/IP networks simultaneously. As shown in figure 8, this new architecture could manage hybrid OSI and Internet-based networks [21]. We named this new architecture SUL (Sub-layer between Upper and Lower layers). This model means: 
International Journal of Computer Networks \& Communications (IJCNC) Vol.9, No.4, July 2017

- There is sub-layer that CMIP could manage both OSI and TCP/UDP/IP networks.

- This sub-layer makes it possible to manage both OSI and TCP/UDP/IP networks with SNMP.

- Some parts of operation carry out with CMIP and other parts with SNMP.

To manage network, protocol selection is very important and depend on the different parameters such as size of network, network traffic, access to the resources and essential management functions. Table 8 compares the management functional areas of CMIP and SNMP. To implement SNMP on the OSI model, a "snmp" selector defines. This selector is used for management and "snmp trap" will utilize to declare alarm. SNMP entity of the manager or agent should listen to this selector for detection the type of message.

To put CMIP on the TCP/UDP/IP network in the OSI model, Low Presentation Protocol (LPP) is proposed for connection TCP/UDP/IP to the upper layers. The LPP in the sub-layer will provide necessary functions of CMIP on the stack protocol TCP/UDP/IP. Also LPP give the required function for SNMP on the OSI protocol stack.

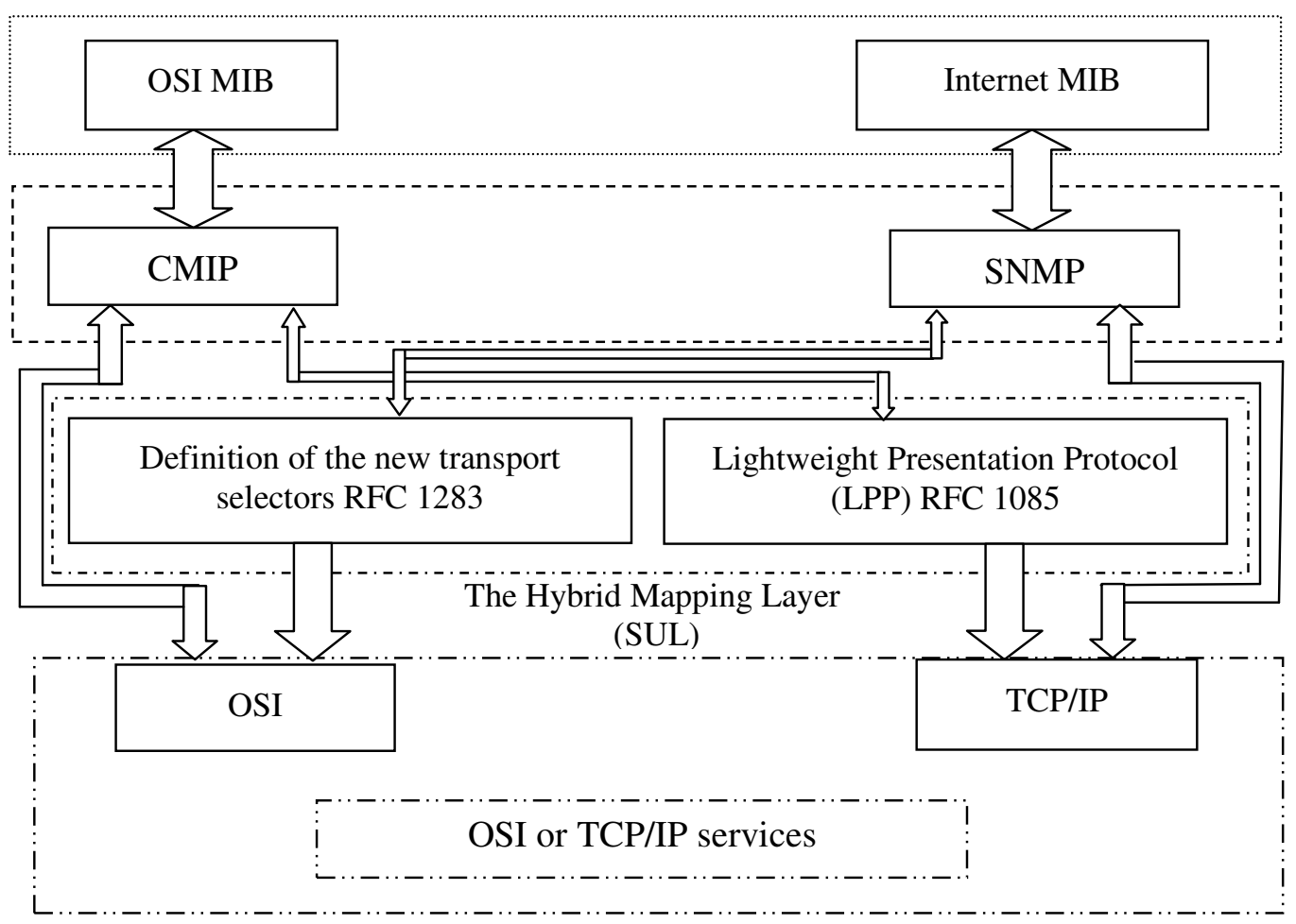

Figure 8. Proposed architecture of network management. 
International Journal of Computer Networks \& Communications (IJCNC) Vol.9, No.4, July 2017

Table 8. Functional management of SNMP and CMIP

\begin{tabular}{|c|c|c|c|c|}
\hline \multicolumn{3}{|c|}{ Areas of Management Functional } & SNMP & CMIP \\
\hline \multicolumn{3}{|c|}{ Configuration Management } & $\mathrm{X}$ & $\mathrm{X}$ \\
\hline \multirow{3}{*}{\multicolumn{2}{|c|}{ Fault Management }} & Detecting and reporting faults & & $\mathrm{X}$ \\
\hline & & Diagnosing fault & $X$ & \\
\hline & & Correcting fault & $\mathrm{X}$ & \\
\hline \multicolumn{3}{|c|}{ Security Management } & & $\mathrm{X}$ \\
\hline \multirow{4}{*}{$\begin{array}{l}\text { Performance } \\
\text { Management }\end{array}$} & \multicolumn{2}{|c|}{ Monitoring performance } & & $\mathrm{X}$ \\
\hline & \multicolumn{2}{|c|}{ Tuning and controlling performance } & $\mathrm{X}$ & \\
\hline & \multicolumn{2}{|c|}{ Reporting on performance, monitoring, tuning and tracking } & & $\mathrm{X}$ \\
\hline & \multicolumn{2}{|c|}{ Testing capacity and special conditions } & & $\mathrm{X}$ \\
\hline \multicolumn{3}{|c|}{ Accounting Management } & $\mathrm{X}$ & \\
\hline
\end{tabular}

\subsection{Proposed Algorithm}

Regarding to three different approaches for information transaction between threads named Semaphore, Deadlock and Critical Section, we have used Critical Section approach, it means if there is shared resource between threads and one thread use a shared resource, another thread doesn't have an ability of using shared resource till first thread finish its work with shared resource

Proposed implemented algorithm for lower layers of Q3 interface is shown in figure 9. The hardware specification of implementation for comparison of proposed algorithm with other algorithm is as Table 9.

Table 9. Hardware specification of simulation

\begin{tabular}{|c|c|c|c|c|}
\hline Station type & CPU type & CPU speed & Bus speed & Number \\
\hline Server & Intel Core i7 & $3.2 \mathrm{GHz}$ & $2400 \mathrm{MHz}$ & 1 \\
\hline Client & Celeron dual core & $2.2 \mathrm{GHz}$ & $800 \mathrm{MHz}$ & 20 \\
\hline
\end{tabular}

In our implementation server simulate the control center and 20 clients simulate the users in the TMN. Each instruction for ordinary approach in ordinary switching telecommunication networks requires 86 bit in average for different protocols while with use of proposed algorithm in the Q3 interface each instruction needs only 64 bit. Our management network was implemented with RS232 network and maximum bit rate 11520 bps. As shown in figure 10, proposed method with Q3 interface implementation has overcome to ordinary method in approximately 300 points average with $26.6 \%$ performance improvement that performance was calculated as relative bit per second improvement for each number of instructions per second. 
International Journal of Computer Networks \& Communications (IJCNC) Vol.9, No.4, July 2017

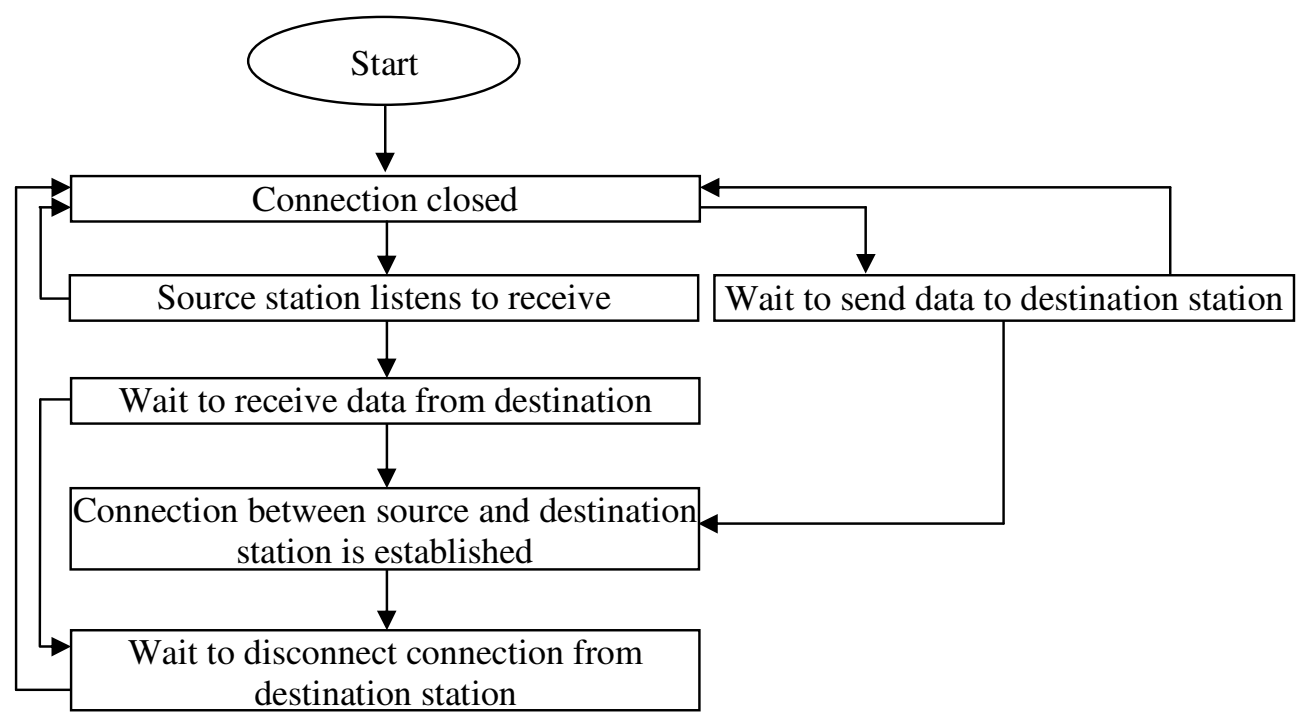

Figure 9. General proposed algorithm to transmit data between client and server

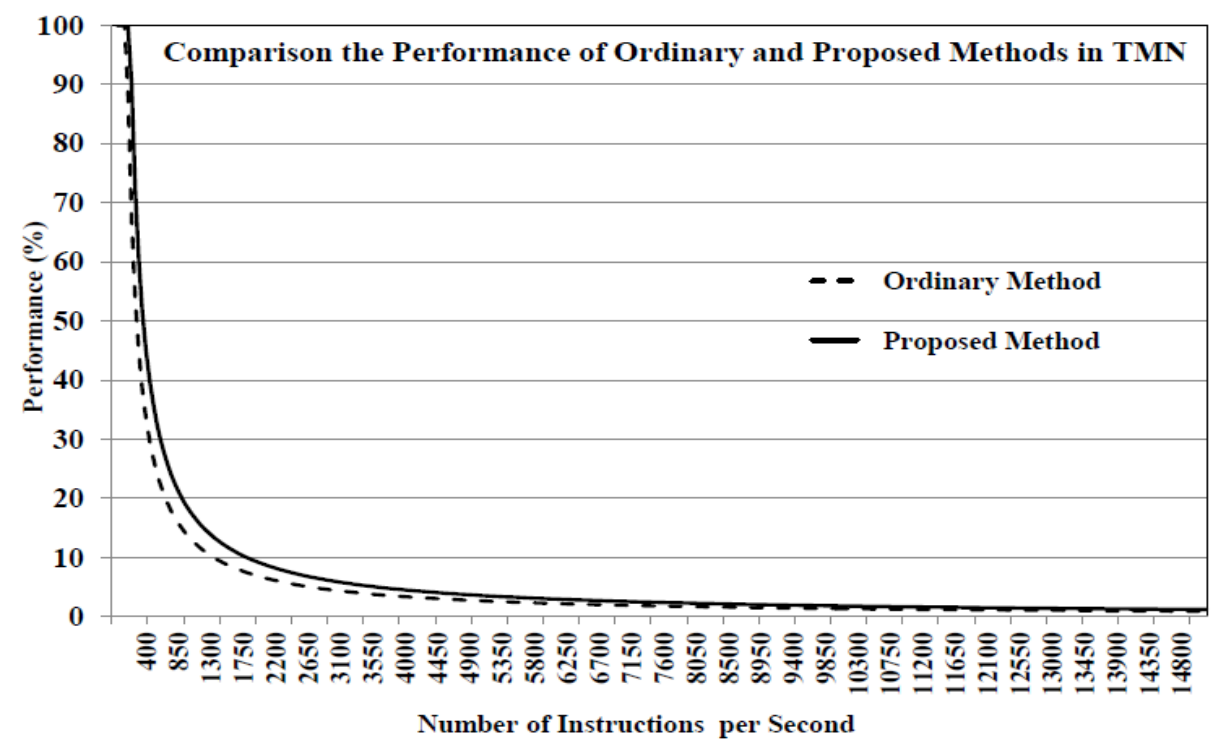

Figure 10. Comparison of the ordinary and proposed methods (Relative improvement for number of instructions per second)

Also to asset performance of the new proposed architecture, first the passing time for receiving response after sending a request is calculated. Calculation of time for three M-Get, M-Set and MEvent Report services is as table 10 [22]. 
International Journal of Computer Networks \& Communications (IJCNC) Vol.9, No.4, July 2017

Table 10. Time calculation for three service M-Get, M-Set and M-Event Report

\begin{tabular}{|l|l|}
\hline \multicolumn{1}{|c|}{ Service Name } & \multicolumn{1}{c|}{ Time calculation procedure } \\
\hline M-Get & $\begin{array}{l}\text { 1. Manager entity will send an M-Get request } \\
\text { 2. Agent entity will response to this request } \\
\text { 3. Passing time will measure between sending request and receiving response }\end{array}$ \\
\hline M-Set & $\begin{array}{l}\text { 1. Manager entity will send an M-Set request } \\
\text { 2. Agent entity will response to this request } \\
\text { 3.The passing time between sending request and receiving response measured }\end{array}$ \\
\hline M-Event Report & $\begin{array}{l}\text { 1. Network simulation software will send an alarm } \\
\text { 2. Agent entity will send an M-Event report to the manager entity } \\
\text { 3. At manager entity, time between consecutive M-Event Report measured }\end{array}$ \\
\hline
\end{tabular}

Figure 11 shows the effectiveness of the proposed architecture (SUL) in comparison to CMIP and SNMP. In figure 11 numbers of operations per second have been calculated and proved that SUI architecture shown in figure 8 has a better performance than CMIP and SNMP. SUL has a $12.5 \%$ performance improvement in M-Get and M-Set services and 25\% in M-Event Report service. For SNMP compare to CMIP, performance improvement is $125 \%$ in M-Get and M-Set services and $66 \%$ in M-Event Report service.

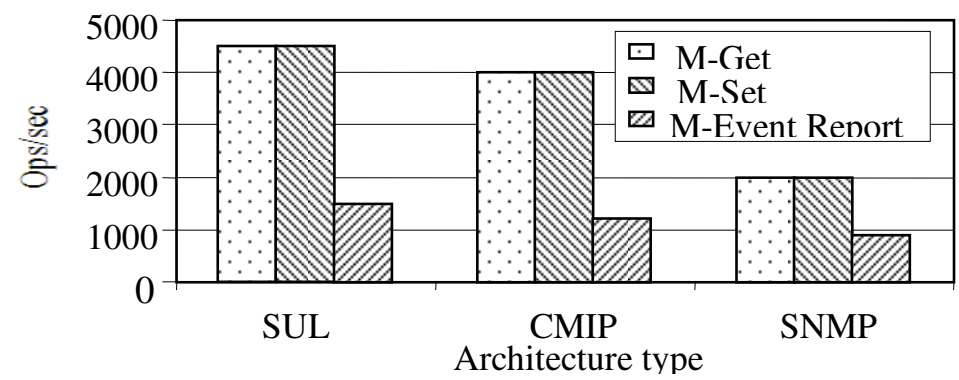

Figure 11. Performance Comparison of SUL (Proposed), CMIP and SNMP models

\section{Conclusions}

In this paper, the layers of Q3 interface was simulated and implemented on laboratory. For management of hybrid OSI and Internet network a new architecture was proposed. The proposed architecture named SUL which is based on the CMIP and SNMP protocols to manage networks. The SUL which is a new sub-layer, stayed between upper layer management protocols and lower layers protocol stacks OSI and TCP/UDP/IP. With use of this sub-layer, CMIP and SNMP protocols will provide required services for users independent of lower layers protocols in OSI and internet models. 
International Journal of Computer Networks \& Communications (IJCNC) Vol.9, No.4, July 2017

This SUL architecture has been proposed to communicate between manager and agent entities fast with lower layers independency. SUL performance will overcome to each CMIP and SNMP performances. Simulation results show the performance improvement of SUL in comparison to CMIP and SNMP protocols in three services M-Get, M-Set and M-Event Report. Also proposed algorithm with Q3 interface has $26.6 \%$ performance improvement averagely in comparison to ordinary method in the TMN to manage all telecommunication switches.

\section{REFERENCES}

[1] King A. \& Hunt R., (2000), "Protocols and architecture for managing TCP/IP network infrastructures", Computer Communications, Vol. 23, pp 1558-1572.

[2] Lakshmi, G. R., (1999), Fundamental of Telecommunication Network Management, IEEE press, New York.

[3] RFC 1189, ITU-T Recommendation X.711, (1990), Common Management Information Services and Protocols for the Internet (CMOT and CMIP).

[4] Sahin, V., Omidyar C.G., \& Bauman T.M., (1988), "Telecommunications management network (TMN) architecture and interworking designs', IEEE Journal on Selected Areas in Communications, Vol. 6, pp 685-696.

[5] ITU-T, M3010, (1996), Principles for a Telecommunications Management Network.

[6] ITU-T, M3020, (2000), TMN Interface Specification Methodology.

[7] Mark, A. \& Miller, P. E., (1992), Trouble Shooting TCP/IP, Prentice Hall.

[8] Pouffer, Y., (1997), ISO Transport Service on top of TCP (ITOT), RFC2126, Network Working Group, Digital Equipment Corporation.

[9] RFC791, (1981), Internet Protocol, Information Sciences Institute University of southern California.

[10] Thomas S. A., (1996), Ipng and TCP/IP protocols, John Wiley \& Sons Inc.

[11] M. Sailaja \& Y. N. Kumar, (2015), "Design and Implementation of Next Generation Networks: A Future Communication System over IP Platform", International Journal of Advanced Research in Electrical, Electronics and Instrumentation Engineering, Vol. 4, No. 11, pp 8634-8638.

[12] V. SURESH, (2016), "Network Management for Next Generation Networks", International Research Journal of Computer Science (IRJCS), Vol. 3, No. 12, pp 45-47.

[13] ITU-T, M.3000, (1995), Maintenance Telecommunications Management Network: Overview of TMN Recommendations.

[14] ITU-T, Q.812, (1997), Upper Layer Protocol Profiles for the Q3 and X Interfaces.

[15] ITU-T, Q.811, (1997), Lower Layer Protocol Profiles for the Q3 and X Interfaces.

[16] Forouzan B., (2009), TCP/IP Protocol Suite, Mcgraw-Hill, 4th edition.

[17] Rose M. T. \& McCloghrie K., (1995), How to Manage Your Network Using SNMP, Prentice Hall.

[18] Duffy J., (2008), Concurrent Programming on Windows, Addison-Wesley, ISBN : 9780321434821.

[19] Schildt H., (2004), The Art of C++, McGraw-Hill/Osborne.

[20] Petzold C., (1998), Programming Windows, Microsoft Press.

[21] Farahani G., (2012), "Design and Implementation of New Architecture to Improve Management Performance of IP-based Networks", International Conference on Information and Computer Networks, Singapore.

[22] Farahani G., (2012), "Design and Implementation of Lower Layers of Q3 Interface in Telecommunication Management Network", Proceedings of the International Network Conference, Port Elizabeth, South Africa. 
International Journal of Computer Networks \& Communications (IJCNC) Vol.9, No.4, July 2017

\section{AUTHOR}

Gholamreza Farahani received his BSc degree in electrical engineering from Sharif University of Technology, Tehran, Iran, in 1998 and MSc and PhD degrees in electrical engineering from Amirkabir University of Technology (Polytechnic), Tehran, Iran in 2000 and 2006 respectively. Currently, he is an assistant professor in the Institute of Electrical and Information Technology, Iranian Research Organization for Science and Technology (IROST), Iran. His research interest is signal processing and computer network especially architecture and protocols.

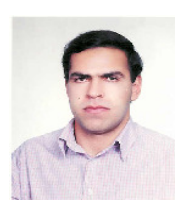

